\title{
POLISH GROUP ACTIONS AND EFFECTIVITY
}

\author{
BARBARA MAJCHER-IWANOW
}

\begin{abstract}
We extend the result of Nadel describing the relationship between approximations of canonical Scott sentences and admissible sets to the general case of orbit equivalence relations induced on an arbitrary Polish space by a Polish group action.
\end{abstract}

Keywords: Polish G-spaces, Scott analysis, recursion, admissible sets

Classification: 03E15, 03C70

\section{INTRODUCTION}

In the paper we extend the following result of Nadel (see [10]) to the general case of Polish $G$-spaces.

Theorem(Nadel) Let $L$ be a countable language, $\mathbb{A}$ be an admissible set and $\mathfrak{M}$ be an L-structure in $\mathbb{A}$. Then for any L-structure $\mathfrak{N}$, if $\mathfrak{M}$ and $\mathfrak{N}$ satisfy the same sentences from the admissible fragment $L_{\mathbb{A}}$, then they satisfy the same sentences of quantifier rank $\alpha \leq$ $o(\mathbb{A})$.

The theorem can be formulated in terms of the logic action as follows. Consider a countable relational language $L=\left(R_{i}^{n_{i}}\right)_{i \in I}$. There is an obvious one-to-one correspondence $\mathfrak{M} \rightarrow x_{\mathfrak{M}}$ between the set of all countable $L$-structures and the set $X_{L}=\prod_{i \in I} 2^{\omega^{n_{i}}}$. The set $X_{L}$ equipped with the product topology becomes a Polish space, the space of all $L$-structures on $\omega$ (see Section 2.5 in [4] or Section 2.D of 2] for details). The group $S_{\infty}$ of all permutations of $\omega$ has the natural continuous action on the space $X_{L}$. It is called the logic action of $S_{\infty}$ on $X_{L}$. Given a structure $x \in X_{L}$, the orbit of $x$ under the logic action consists of all structures isomorphic to $x$. Now the Nadel's theorem reads as follows. 
Let $\mathbb{A}$ be an admissible set and $L \in \mathbb{A}$ be a countable relational language. Let $x, y \in X_{L}$ and $x \in \mathbb{A}$. Then if $x$ and $y$ are in the same invariant Borel sets of the form $B_{\sigma}=\left\{z \in X_{L}: z \models \sigma\right\}$ for some $\sigma \in L_{\mathbb{A}}$, then for every $\alpha \leq o(\mathbb{A}), x$ and $y$ are in the same invariant $\Pi_{\alpha}^{0}$-subsets of $X_{L}$.

The theorem can be viewed as an assertion to what extent a structure $x$ of a given language $L$ is determined (up to isomorphism) by the set of sentences from $L_{\mathbb{A}}$ true in $x$. We shall ask the general question:

Given a Polish $G$-space $X$ which is in some sense coded in an admissible set $\mathbb{A}$ and $x \in X$, to what extent is the orbit $G \cdot x$ determined by the family of those invariant Borel sets containing $x$ which have Borel codes in $\mathbb{A}$ ?

In the paper we give an answer to this question. It generalizes the Nadel's theorem for any Polish group $G$ and any Polish $G$-space $X$. It is formulated in the second part of Section 2. In the first part of this section we settle the parallel question on the ground of effective descriptive set theory. This is the main result of the paper. A simple proof of this theorem applies some tools and facts from the generalized Scott analysis of continuous actions of Polish groups on Polish spaces developed by Hjorth in [6].

\section{Preliminaries}

In the first part of this section we recall standard notation and facts concerning Polish group actions as well as the brief description of Hjorth method. In the second one we give a summary introduction to effective descriptive set theory.

1.1. Notation. A Polish space (group) is a separable, completely metrizable topological space (group). We shall write $(X, d)$ if $d$ is a compatible metric for $X$. If a Polish group $G$ continuously acts on a Polish space $X$, then we say that $X$ is a Polish $G$-space. We say that a subset of $X$ is invariant if it is $G$-invariant. All basic facts concerning Polish $G$-spaces can be found in [4, 6] and [7].

The Vaught $*$-transform of a set $B \subseteq X$ with respect to an open $H \subseteq G$ is the set $B^{* H}=\{x \in X:\{g \in H: g x \in B\}$ is comeagre in $H\}$. A set $B$ is invariant if and only if $B^{* G}=B$.

Definition A (Hjorth) Let $x, y \in X$. For basic open $V, W \subseteq G$ we define the relation $(y, V) \leq_{\alpha}(x, W)$ by simultaneous induction on the ordinal $\alpha$. 


$$
\begin{aligned}
& (y, V) \leq_{1}(x, W) \text { if } \overline{V \cdot y} \subseteq \overline{W \cdot x} \\
& (y, V) \leq_{\alpha+1}(x, W) \text { if for every basic open } V^{\prime} \subseteq V \\
& \text { there is a basic open } W^{\prime} \subseteq W \text { such that }\left(x, W^{\prime}\right) \leq_{\alpha}\left(y, V^{\prime}\right) \\
& (y, V) \leq_{\lambda}(x, W) \text { if }(y, V) \leq_{\alpha}(x, W) \text { for every } \alpha<\lambda \\
& \text { if } \lambda \text { is limit. }
\end{aligned}
$$

It is shown in [6] that the relation $\leq_{\alpha}$ is transitive and $(y, V) \leq_{\alpha}(x, W)$ implies $(y, V) \leq_{\beta}(x, W)$, whenever $\beta \leq \alpha$. Moreover for every ordinal $\alpha$ and $x \in X$ we have $(x, G) \leq_{\alpha}\left(x^{\prime}, G\right)$ whenever $x^{\prime} \in G \cdot x$. For our purpose the most important property is as follows.

Lemma $\mathbf{B}$ (Hjorth) Let $x, y \in X, V, W \subseteq G$ be basic open sets and $\alpha$ be a countable ordinal such that $(y, V) \leq_{\alpha}(x, W)$. Then for every $\Pi_{\alpha}^{0}$-set $B \subseteq X$ if $x \in B^{* W}$, then $y \in B^{* V}$.

\subsection{Review of basic notions and facts from effective descriptive set the-}

ory. We assume here acquaintance with rudiments of recursion theory. We recall only those notions and facts we shall use in the paper. The review below is based on [8] and [9] (Chapters 3,7), where one can find a complete exposition.

We shall consider merely recursively presented Polish spaces. A Polish space $(X, d)$ is recursively presented if it is endowed with a recursive presentation, i.e. a sequence $\left\{r_{i}: i \in \omega\right\}$ densely contained in $X$ and such that the $(i, j, k, m)$-relations $d\left(r_{i}, r_{j}\right) \leq m / k+1$ and $d\left(r_{i}, r_{j}\right)<m / k+1$ are recursive. The class of recursively presented spaces includes $\omega$, the Baire space $\mathcal{N}$, the Cantor space $\mathcal{C}$, the reals $\mathcal{R}$ and it is closed under finite products.

If $X$ is a recursively presented space with a fixed recursive presentation, then one can naturally define the canonical basis of open neighbourhoods of $X$, an effective enumeration $\left\{U_{n}^{X}: n \in \omega\right\}$ of the basis and a ternary recursive function $g(k, l, m)$ with $\operatorname{Dom}(g)=\omega^{3}$ so that

$$
U_{k}^{X} \cap U_{l}^{X}=\bigcup_{m \in \omega} U_{g(k, l, m)}^{X}
$$

We call a set $A \subseteq X$ semirecursive or effectively open if there is a recursive function $s: \omega \rightarrow \omega$ such that $A=\bigcup_{n} U_{s(n)}^{X}$.

The pointclass of semirecursive pointsets (of recursively presented spaces) is denoted by $\Sigma_{1}^{0}$ and $\Pi_{1}^{0}$ stand for the pointclass of effectively closed (i.e. complements of 
semirecursive) pointsets. The ambiguous class $\Delta_{1}^{0}=\Sigma_{1}^{0} \cap \Pi_{1}^{0} 1$ consists of recursive pointsets. The pointclass $\Delta_{1}^{0}$ contains the empty set, every product space, every recursive relation on $\omega^{k}$, every basic neighbourhood $U_{n}^{X}$ and the basic neighbourhood relation $\left\{(x, n): x \in U_{n}^{X}\right\}$ for each $X$.

If we start from the pointclass $\Sigma_{1}^{0}$ of effectively open sets and $\omega_{1}^{C K}$-times alternately take the operation of complementation and the operation of "effective countable unions" (the Church-Kleene ordinal $\omega_{1}^{C K}$ is the first non-recursive ordinal), then we obtain the pointclass

$$
H Y P=\bigcup_{\zeta<\omega_{1}^{C K}} \Sigma_{\zeta}^{0}
$$

of hyperarithmetic pointsets, the effective analogue of the pointclass of Borel pointsets. On the other hand if we apply to $\Sigma_{1}^{0}$ the operations of complementation and projection along Baire space $\mathcal{N}$, then we build the pointclasses

$$
\Sigma_{1}^{1}=\exists^{\mathcal{N}} \Pi_{1}^{0}, \Pi_{1}^{1}=\neg \Sigma_{1}^{1} \text { and } \Delta_{1}^{1}=\Sigma_{1}^{1} \cap \Pi_{1}^{1} .
$$

The following statement is true for every recursively presented space $X$.

Theorem C (The Suslin-Kleene theorem) For every $A \subseteq X$ we have

$$
A \in H Y P \text { iff } A \in \Delta_{1}^{1} \text {. }
$$

Definition D Let $X, Y$ be recursively presented spaces with bases $\left\{U_{n}^{X}\right\}$ and $\left\{U_{n}^{Y}\right\}$ respectively, $\Gamma=\Sigma_{\xi}^{0}, \Sigma_{1}^{1}$ or $\Delta_{1}^{1}$.

(1) A function $f: X \rightarrow Y$ is $\Gamma$-recursive if $\left\{(x, n): f(x) \in U_{n}^{Y}\right\} \in \Gamma$.

(2) A partial function $f: X \rightarrow Y$ is $\Gamma$-recursive on its domain if there is some $P \subseteq X \times \omega$ such that $P \in \Gamma$ and $\left\{(x, n): f(x) \in U_{n}^{Y}\right\}=P \cap(\operatorname{dom}(f) \times \omega)$.

(3) An element $x \in X$ is $\Gamma$-recursive if $\left\{n: x \in U_{n}^{X}\right\} \in \Gamma$.

$\Sigma_{1}^{0}$-recursive functions (points) are simply called recursive and $\Delta_{1}^{1}$-recursive hyperarithmetic. The pointclass $\Delta_{1}^{1}$ satisfies the following closure properties.

Theorem $\mathbf{E}$ The pointclass $\Delta_{1}^{1}$ is closed under $\vee, \wedge$, negation, existential and universal quantification over $\omega$ and substitution of $\Delta_{1}^{1}$-recursive functions 2 .

\footnotetext{
${ }^{1}$ notice using lightface font for distinguishing effective classes from their non-effective analogues printed later on in boldface

${ }^{2}$ This means that for every partial $\Delta_{1}^{1}$-recursive function $f: X \rightarrow Y$ and every $\Delta_{1}^{1}$-set $B \subseteq Y$ there is a $\Delta_{1}^{1}$-set $A \subseteq X$ such that $f^{-1}[B]=A \cap \operatorname{dom}(f)$.
} 
For every space $Z$, an element $z \in Z$ and a pointclass $\Gamma=\Sigma_{\xi}^{0}, \Pi_{\xi}^{0}, \Sigma_{1}^{1}$ or $\Pi_{1}^{1}$ we shall consider also the relativized pointclass $\Gamma(z)$ : a set $A \subseteq X$ is in $\Gamma(z)$ if there is some $Q \subseteq Z \times X$ such that $Q \in \Gamma$ and $A=Q_{z}$, where $Q_{z}=\{x \in X$ : $(z, x) \in Q\}$. Then we define the ambiguous classes $\Delta_{1}^{0}(z)=\Sigma_{1}^{0}(z) \cap \Pi_{1}^{0}(z)$ and $\Delta_{1}^{1}(z)=\Sigma_{1}^{1}(z) \cap \Pi_{1}^{1}(z)$. The sets in $\Sigma_{1}^{0}(z)$ are called semirecursive in $z$, the sets in $\Delta_{1}^{1}(z)$ - hyperarithmetic in $z$.

In the obvious way we may relativize the notions of recursive and hyperarithmetic functions. It should be mentioned that the relativized versions of the theorems cited above remain true.

It is easy to see that a subset of a given space $X$ is open if and only if it is semirecursive in some $z \in \mathcal{N}$. In fact for every recursively presented space $X$ there is a semirecursive $P \subseteq \mathcal{N} \times X$ (called a good parametrization system) which is universal for the pointclass of open subsets of $X$ so that for every $A \subseteq X$ we have

$$
A \in \Sigma_{1}^{0} \text { iff } A=P_{\epsilon} \text { for a recursive } \epsilon \in \mathcal{N} 3
$$

Starting with such a good parametrization system $P \subseteq \mathcal{N} \times \omega \times \omega$ for subsets of $\omega \times \omega$ we can define some special coding of Borel sets by elements of $\mathcal{N}$. This method is described in detail in 9] (Chapter 7.A), we shall only recall some notation and properties

Using $P$ we define by recursion on the countable ordinal $\xi$ an increasing family $\left\{B C_{\xi}\right\}_{\xi}$ of subset of $\mathcal{N}$ with the union $B C=\bigcup_{\xi} B C_{\xi}$ - the set of Borel codes.

Then for every recursively presented space $X$ we can define a coding function $\pi: B C \rightarrow \operatorname{Borel}(X)$ so that $\left.\pi\right|_{B C_{\xi}}: B C_{\xi} \rightarrow \boldsymbol{\Sigma}_{\xi}^{0}(X)$ is "onto", for every countable $\xi$. Given $A \in \operatorname{Borel}(X)$ we say that an irrational $\alpha \in B C$ is a Borel code of $A$ whenever $\pi(\alpha)=A$. This coding function has the following important property.

Lemma $\mathbf{F}$ Let $z \in \mathcal{N}$ and $A \subseteq X$. Then $A$ is hyperarithmetic in $z$ if and only if it has a Borel code recursive in $z$.

\section{General versions of the Nadel's theorem}

The section is divided into two parts. In the first one we shall prove an effective counterpart of the Nadel's theorem. In the second part we translate these results into the language of admissible sets.

\footnotetext{
${ }^{3}$ This is a part of The Good Parametrization Lemma, see [9] 3H.1
} 
2.1. Hyperarithmetic actions and Borel sets. From now on we shall always assume that a Polish group $G$ is a recursively presented space and $\left\{\hat{g}_{i}: i \in \omega\right\}$ is a fixed recursive presentation of $G$. Let $\left\{V_{i}: i \in \omega\right\}$ be an effective enumeration of the canonical basis of $G$. We additionally assume that $V_{0}=G$. A Polish $G$-space $X$ is recursively presented and $\left\{U_{i}: i \in \omega\right\}$ is an effective enumeration of the canonical basis of $X$.

It is proved in [6] that the relation $\left\{(x, y, n, k):\left(x, V_{k}\right) \leq_{\xi}\left(y, V_{n}\right)\right\} \subseteq X^{2} \times \omega^{2}$ is Borel for every countable ordinal $\xi$. The following lemma is an effective version of this statement.

Lemma 1. Let $G, X$ be recursively presented, the group operations of $G$ and the $G$-action on $X$ be hyperarithmetic. Then for every $\xi<\omega_{1}^{C K}$ the set

$$
H_{\xi}=\left\{(x, y, n, k):\left(x, V_{k}\right) \leq_{\xi}\left(y, V_{n}\right)\right\}
$$

is hyperarithmetic.

Proof. First consider $H_{1}=\left\{(y, x, k, n): \overline{V_{k} y} \subseteq \overline{V_{n} x}\right\}$. We have

$$
\begin{gathered}
(y, x, k, n) \in H_{1} \\
\mathbb{\Downarrow} \\
(\forall i)\left(y \in V_{k}^{-1} U_{i} \Rightarrow x \in V_{n}^{-1} U_{i}\right) \\
\mathbb{v} \\
(\forall i)\left((y, x) \in\left[\left(X \backslash V_{k}^{-1} U_{i}\right) \times X\right] \cup\left[V_{k}^{-1} U_{i} \times V_{n}^{-1} U_{i}\right]\right) \\
\mathbb{\Downarrow} \\
(y, x, k, n) \in \bigcap_{i}\left(\left[\left(X \backslash V_{k}^{-1} U_{i}\right) \times X\right] \cup\left[V_{k}^{-1} U_{i} \times V_{n}^{-1} U_{i}\right]\right) \times\{k\} \times\{n\} .
\end{gathered}
$$

Hence $H_{1}=\bigcup_{k, n} \bigcap_{i}\left(\left[\left(X \backslash V_{k}^{-1} U_{i}\right) \times X\right] \cup\left[V_{k}^{-1} U_{i} \times V_{n}^{-1} U_{i}\right]\right) \times\{k\} \times\{n\}$.

Now observe that if $V \subseteq G$ and $U \subseteq X$ are open, then $V U=\bigcup\left\{\hat{g}_{j} U: \hat{g}_{j} \in\right.$ $V, j \in \omega\}$. Indeed, take any $g \in V$ and $x \in U$. It follows from the continuity of the group operations of $G$ and the $G$-action that there are open $g \in W \subseteq V$ and $x \in A \subseteq U$ such that $W W^{-1} W \subseteq V$ and $W^{-1} W A \subseteq U$. Then for any $f \in W$ we have $g \in f W^{-1} W$ and so $g x \in f W^{-1} W A \subseteq f U$. In particular $V_{k}^{-1} U_{i}=$ $\bigcup\left\{\hat{g}_{j}^{-1} U_{i}: \hat{g}_{j} \in V_{k}\right\}$. Since the $G$-action is hyperarithmetic, every element $\hat{g}_{j}$ of the recursive presentation $\left\{\hat{g}_{i}: i \in \omega\right\}$ is recursive and every basic open $U_{i}$ is recursive, then by closeness of $\Delta_{1}^{1}$ pointclass under substitution property of $\Delta_{1}^{1}$-functions, $\hat{g}_{j}^{-1} U_{i} \in H Y P$. Hence by other closure properties from Theorem E, the set $H_{1}$ is hyperarithmetic. 
Next we have

$$
\begin{aligned}
& (y, x, k, n) \in H_{\mu+1} \\
& \Uparrow \\
& (\forall r)(\exists s)\left(V_{r} \subseteq V_{k} \Rightarrow\left(V_{s} \subseteq V_{n}\right) \wedge\left(x, V_{s}\right) \leq_{\mu}\left(y, V_{r}\right)\right) \\
& \mathbb{1} \\
& \left.(\forall r)(\exists s)\left(V_{r} \subseteq V_{k} \Rightarrow\left(V_{s} \subseteq V_{n}\right) \wedge(x, y, s, r) \in H_{\mu}\right)\right) \\
& \Uparrow \\
& (x, y) \in \bigcap_{\substack{r \\
V_{r} \subseteq V_{k}}}\left(\bigcup_{\substack{s \\
V_{s} \subseteq V_{n}}} \pi_{1,2}\left(H_{\mu} \cap\left(X^{2} \times\{r\} \times\{s\}\right)\right)\right) \\
& \Uparrow \\
& (y, x, k, n) \in s\left(\bigcap_{\substack{r \\
V_{r} \subseteq V_{k}}}\left(\bigcup_{V_{s} \subseteq V_{n}} \pi_{1,2}\left(H_{\mu} \cap\left(X^{2} \times\{r\} \times\{s\}\right)\right)\right)\right) \times\{k\} \times\{n\},
\end{aligned}
$$

where $\pi_{1,2}(x, y, r, s)=(x, y)$ and $s(x, y)=(y, x)$.

Since $G$ is recursively presented, the relation $\left\{(k, r): V_{r} \subseteq V_{k}\right\}$ is recursive. Hence using closure properties of $\Delta_{1}^{1}$ we argue that if $H_{\mu}$ is hyperarithmetic, then so is $H_{\mu+1}$.

On the other hand if $\xi$ is a recursive limit ordinal and $H_{\mu}$ is hyperarithmetic for every $\mu<\xi$, then $H_{\xi}=\bigcap_{\mu<\xi} H_{\mu}$ is an "effective intersection" of hyperarithmetic sets - thus is also hyperarithmetic.

Now we are ready to prove the main result of this part.

Theorem 2. Assume that $G, X$ are presented recursively in $\zeta$, the group operations of $G$ and the $G$-action on $X$ are hyperarithmetic in $\zeta$. If $x, y \in X, x$ is hyperarithmetic in $\zeta$ and $x, y$ are in the same invariant sets hyperarithmetic in $\zeta$, then for every $\alpha \leq \omega_{1}^{C K}(\zeta) \quad x, y$ are in the same invariant Borel sets of Borel rank $\alpha$.

Proof. Assume that $\zeta \in \Delta_{1}^{0}$. Let $\alpha<\omega_{1}^{C K}$ and $B \subseteq X$ be an invariant $\Pi_{\alpha}^{0}$-set containing $x$. By the lemma above $H_{\alpha}$ is hyperarithmetic. Since $x$ is hyperarythmetic, then the set $\left\{z \in X:(z, G) \leq_{\alpha}(x, G)\right\}=\pi_{1}\left(H_{\alpha} \cap(X \times\{x\} \times\right.$ $\{0\} \times\{0\})$ is an invariant hyperarithmetic set containing $x$. Thus $y$ is an element of this set, i.e. $(y, G) \leq_{\alpha}(x, G)$. This by Lemma B implies $y \in B$.

Now consider the case $B \in \Pi_{\omega_{1}^{C K}}^{0}$. Since $\omega_{1}^{C K}$ is a limit ordinal, then $B$ is an intersection of $\omega_{1}^{C K}$ invariant sets of Borel rank $\xi<\omega_{1}^{C K}$. If $x \in B$, then $x$ belongs to each of the sets. Hence by the first part of the proof $y \in B$.

In the case of arbitrary $\zeta$ we use the same arguments based on the obvious relativization of Lemma 1 
2.2. General version of the Nadel's theorem. We assume that the reader is familiar with the most basic notions of admissible sets. Any necessary background can be easily provided by [1] and [5].

We only remind the reader that an admissible set $\mathbb{A}$ is a transitive model of KPU, in the sense of 1]. Such models are considered as two-sorted structures of some language $L$ with symbols $\emptyset, \in$, where one of the sorts corresponds to urelements and usually forms a relational first-order structure with respect to the symbols of $L$ distinct from $\emptyset$ and $\in$. Here we assume that $\mathbb{A}$ satisfies KPU with respect to all formulas of $L$ ( $\mathbb{A}$ is admissible with respect to $L$ in terms of [10]).

As we recalled in Section 1.2 for every recursively presented space $X$ we can define a partial function $\pi: \mathcal{N} \stackrel{\text { onto }}{\rightarrow} \operatorname{Borel}(X)$ with domain the set $B C$ of Borel codes, so that a set $B \subseteq X$ is hyperarithmetic (resp. hyperarithmetic in $z$ ) if and only if it has a recursive (resp. recursive in $z$ ) Borel code. Thus we can discuss Borel sets in terms of their Borel codes. To do that in an admissible set $\mathbb{A}$ we shall assume that $\mathbb{A}$ contains some countable set (possibly as a set of urelements). We will say that $\omega$ is realizable in an admissible set $\mathbb{A}$ if the set contains a copy of the structure $\langle\omega,<\rangle$ as an element. Observe that $\omega$ is realizable in any admissible set satisfying Infinity Axiom. On the other hand the $\omega$-model $\mathbf{H F}(\omega,<)$ does not realize $\omega$. Since it does not cause any misunderstanding, we shall write $\omega$ even if we work not with $\omega$ itself but with its copy.

Definition 3. Let $G$ be a recursively presented group with a basis $\left\{V_{m}: m \in \omega\right\}$ and $X$ be a recursively presented Polish $G$-space with a basis $\left\{U_{n}: n \in \omega\right\}$. Let $\mathbb{A}$ be an admissible set such that $\omega$ is realizable in $\mathbb{A}$.

(1) We say that $x \in X$ is codable in $\mathbb{A}$ if the set $r_{x}=\left\{n: x \in U_{n}\right\}$ is an element of $\mathbb{A}$.

(2) We say that the group $G$ is codable in $\mathbb{A}$ if the relations $r_{o}=\{(k, l, n)$ : $\left.V_{k} V_{l} \subseteq V_{n}\right\}$ and $r_{i}=\left\{(k, l): V_{k}^{-1} \subseteq V_{l}\right\}$ are in $\mathbb{A}$.

(3) We say that the $G$-action on $X$ is codable in $\mathbb{A}$ if the relation $r_{a}=\{(k, i, j)$ : $\left.V_{k} U_{i} \subseteq U_{j}\right\}$ is an element of $\mathbb{A}$.

Theorem 4. Let $\mathbb{A}$ be an admissible set realizing $\omega$. Let $G, X$ and $x \in X$ be codable in $\mathbb{A}$. Then for any $y \in X$ if $x$ and $y$ are in the same invariant Borel sets with Borel codes belonging to $\mathbb{A}$, then they are in the same invariant Borel sets of Borel rank $\alpha \leq o(\mathbb{A})$.

Proof. Let $r_{x}, r_{o}, r_{i}, r_{a}$ have the same meaning as in Definition 3, Then easily $x$, the group operations of $G$ and the $G$-action on $X$ are recursive in $r_{x}, r_{o}, r_{i}$ and 
$r_{a}$ respectively. For example, since $\left\{(g, x, j): g \cdot x \in U_{j}, j \in \omega\right\}=\bigcup\left\{V_{k} \times U_{i} \times\{j\}\right.$ : $\left.(k, i, j) \in r_{a}\right\}$, then the $G$-action on $X$ is recursive in $r_{a}$.

Let $\alpha<o(\mathbb{A})$ be a countable ordinal. We can find $z \in \mathbb{A}$ such that $r_{x}, r_{o}, r_{i}, r_{a}$ are hyperarithmetic in $z$ and $\alpha<\omega_{1}^{C K}(z)$. By The Suslin-Kleene Theorem every set which is hyperarithmetic in $z$ has a recursive in $z$ Borel code. Then by the assumptions $x$ and $y$ are in the same invariant Borel sets which are hyperarithmetic in $z$. Hence we can apply Theorem 2 to see that any invariant $\Pi_{\alpha}^{0}$-set $B \subseteq X$ containing $x$ contains $y$.

To finish the proof notice that each invariant $\Pi_{o(\mathbb{A})^{-s e t}}^{0} \subseteq X$ is an intersection of a family $o(\mathbb{A})$-many invariant Borel sets of Borel ranks $\xi<o(\mathbb{A})$. If $x$ belongs to such a $B$, then $y \in B$ by the first part of the proof.

At the end we shall see that Theorem 4 indeed generalizes the theorem of Nadel quoted in Introduction. We deal with the translation of this theorem into the language of the logic actions. The set of all finitary permutations is a dense countable subgroup which can be recursively enumerated and turned into a recursive presentation of $S_{\infty}$. Similarly the space $X_{L}$ can be recursively presented by an appropriate recursive enumeration of the set of all ultimately equal zero sequences. Moreover the logic action on $X_{L}$ is a $\left(\Sigma_{1}^{0}\right.$-)recursive function.

Let $\mathbb{A}$ be an admissible set and $\mathfrak{M} \in \mathbb{A}$ be an $L$-structure. Then easily $S_{\infty}$, the logic action of $S_{\infty}$ on $X_{L}$ and $x_{\mathfrak{M}}$ are codable in $\mathbb{A}$ in the sense of Definition 3 ,

To every sentence $\sigma \in L_{\omega_{1} \omega}$ we can assign the invariant set $B_{\sigma}=\left\{x_{\mathfrak{M}} \in X_{L}\right.$ : $\mathfrak{M} \models \sigma$ \}. Moreover for each Borel $\boldsymbol{\Sigma}_{\alpha}^{0}$-set $B$ invariant under the logic action there is an $L_{\omega_{1} \omega}$-sentence $\sigma$ of quantifier rank $\alpha$ (the theorem of Lopez-Escobar, see [1] and [7, Theorem 16.8 and its proof) such that $B=B_{\sigma}$. Finally we see that $B$ has a Borel code in $\mathbb{A}$ if and only if $B=B_{\sigma}$ for some $\sigma \in L_{\mathbb{A}}$.

\section{REFERENCES}

[1] J.Barwise, Admissible Sets and Structures, Springer-Verlag, NY, 1975.

[2] H.Becker, Polish group actions: Dichotomies and generalized elementary embeddings, J. Amer. Math. Soc. 11, 397 - 449 (1998).

[3] H.Becker, Topics in invariant descriptive set theory, Annals of Pure and Appl. Logic, 111, 145 - 184 (2001).

[4] H.Becker and A.Kechris, The Descriptive Set Theory of Polish Group Actions, Cambridge University Press, Cambridge, 1996.

[5] Yu.Ershov, Definability and Computability, Consultants Bureau, NY, 1996.

[6] G.Hjorth, Classification and Orbit Equivalence Relations. AMS 1991

[7] A.Kechris, Classical Descriptive Set Theory, Springer-Verlag, New York, 1995. 
[8] A.S. Kechris and D.A. Martin, Infinite game and effective descriptive set theory, Analytic sets, Academic Press 403-470, 1980

[9] Y.N.Moschovakis, Descriptive Set Theory, Studies in Logic (North Holland, Amsterdam 1980)

[10] M.Nadel, Scott sentences and admissible sets, Ann. Math. Log. 7(1974), 269 - 294.

[11] R.Sami, Polish group actions and the Vaught conjecture, Trans. Amer. Math. Soc., 341, 335 - 353 (1994).

Institute of Mathematics, University of Wrocław, pl.Grunwaldzki 2/4, 50-384 Wrocław, Poland

E-mail: ivanov@math.uni.wroc.pl 